\title{
Complementing the Lagrangian Density of the E. M. Field and the Surface Integral of the p-v Vector Product
}

\author{
Mirwais Rashid \\ Delft University of Technology, Delft, The Netherlands \\ E-mail: mirwaisrashid@hotmail.com \\ Received October 13, 2010; revised December 5, 2010; accepted December 9, 2010
}

\begin{abstract}
Considering the Lagrangian density of the electromagnetic field, a $4 \times 4$ transformation matrix is found which can be used to include two of the symmetrized Maxwell's equations as one of the Euler-Lagrange equations of the complete Lagrangian density. The $4 \times 4$ transformation matrix introduces newly defined vector products. In a Theorem the surface integral of one of the newly defined vector products is shown to be reduced to a line integral.
\end{abstract}

Keywords: Electromagnetic Field, Parallel-Vertical Product, Surface Integral

\section{Introduction}

In physics there are four equations which are known as the Maxwell's equations. The two of these equations are the Gauss's law for the electric field and the Gauss's law for the magnetic field and the other two are the Ampère's law and the Faraday's law [1]. In the theory for the massless electromagnetic vector field [2] the Gauss's law for the electric field and the Ampère's law are written in a succinct form as Equation (1) below and the Lagrangian density is found for this Equation (1). However, the Gauss's law for the magnetic field and Faraday's law are omitted apparently because of the fact that there are no magnetic charges detected yet. In this article the Faraday's law and the Gauss's law for the magnetic field are formulated as one tensor Equation (8) and the Lagrangian density is written for Equation (8). Even if there are no magnetic charges the Lagrangian density of the two omitted Maxwell's equations which contains the difference between the potential energy density and the kinetic energy density of the electromagnetic field [2] should not be omitted since the Ampère's law alone does not imply electromagnetic waves and it is the Ampère's law together with Faraday's law which among others implied the electromagnetic waves and there is therefore a need to complement the existing Lagrangian density [2]. Reference [1] gives the Maxwell's equations in the integral form, however, using the existing mathematical theorems the Maxwell's equations can be written in differential form [3]. In this article the relation between the tensor of
Equation (1) and the tensor of Equation (8) is found as a $4 \times 4$ matrix the components of which contain newly defined vector products which are called here as the parallel-vertical (abbreviated as the p-v) vector product and the parallel-horizontal vector product. In finding the Lagrangian from the Lagrangian density one should integrate over the volume concerned.

In this article the surface integral [4] of the parallelvertical vector product is shown to be reducible to a line integral.

\section{The Maxwell's Equations in Tensor Forms}

The four Maxwell's equations are written with tensor notations in more compact forms as the following two Equations [2]

$$
\begin{gathered}
\partial_{\mu} F^{\mu v}=j^{v} \\
\partial_{\alpha} F_{\beta \gamma}+\partial_{\beta} F_{\gamma \alpha}+\partial_{\gamma} F_{\alpha \beta}=0
\end{gathered}
$$

with

$$
F^{\mu v}=\left[\begin{array}{cccc}
0 & -E_{1} & -E_{2} & -E_{3} \\
E_{1} & 0 & -B_{3} & B_{2} \\
E_{2} & B_{3} & 0 & -B_{1} \\
E_{3} & -B_{2} & B_{1} & 0
\end{array}\right]
$$

and

$$
j^{v}=\left(\rho, J_{1}, J_{2}, J_{3}\right)
$$




$$
\partial_{\mu}=\left(\frac{\partial}{\partial t}, \frac{\partial}{\partial x}, \frac{\partial}{\partial y}, \frac{\partial}{\partial z}\right)
$$

The Lagrangian density which leads to Equation (1) as its Euler-Lagrange Equation of motion [5], is the following

$$
\mathcal{L}_{1}=-\frac{1}{4} F_{\mu v} F^{\mu v}-j^{v} A_{v}
$$

with $A$ being the vector potential with four components, the first component of which is the electrical po- tential, and the three other components of which are the components of the three dimensional vector potential $\mathbf{A}$, the curl of which is the magnetic field $\mathbf{B}$.

However, Equation (6) which is well known in the published literature does not lead to Equation (2) directly as its Euler-Lagrange equation of motion.

To write the complete Lagrangian density of the Maxwell's equations withterms for eventual magnetic monopoles, Equation (2) is written in a modified form as follows

$$
\left(\frac{\partial}{\partial t},-\frac{\partial}{\partial x},-\frac{\partial}{\partial y},-\frac{\partial}{\partial z}\right)\left[\begin{array}{cccc}
0 & B_{1} & B_{2} & B_{3} \\
-B_{1} & 0 & E_{3} & -E_{2} \\
-B_{2} & -E_{3} & 0 & E_{1} \\
-B_{3} & E_{2} & -E_{1} & 0
\end{array}\right]=\left(\zeta,-Y_{1},-Y_{2},-Y_{3}\right)
$$

Equations (1) and (2) apparently seem to be less compact than the Maxwell's equations in the differential-form as presented in the article of the Journal PIER, mentioned here as reference [6]. However, Equation (2) can be made to have a compacter form than its presented form of Equation (2) with the tensor notation, as its symmetrized form is shown as Equation (5). Equation (2) can be obtained from Equation (7) when the vector $y_{v}=\left(\zeta,-Y_{1},-Y_{2},-Y_{3}\right)$ on the right hand side of Equation (7) is set to be equal to zero. Denoting the $4 \times 4$ ma- trix in Equation (7), as $K_{\theta_{v}}$, one may write Equation (7) in analogy with Equation (1) as follows

$$
\partial^{\theta} K_{\theta v}=y_{v}
$$

One may presume, firstly, a transformation to exist which changes $F_{\mu v}$ into $K_{\theta v}$ as follows

$$
K_{\theta v}=T_{\theta \mu} F_{\mu v}
$$

then a transformation matrix of the following form is found

$$
\begin{aligned}
& (\mathbf{E} \times \mathbf{B})_{2} \\
& (\mathbf{E} \underset{\times}{\Rightarrow} \mathbf{B})_{3} \quad(\mathbf{E} \underset{\times}{\Rightarrow} \mathbf{B})_{2} \\
& B_{2}^{2}-(\widetilde{\mathbf{E} \times \mathbf{E}})_{2} \quad(\underset{\times}{\Rightarrow} \mathbf{B})_{1} \\
& (\mathbf{E} \underset{\times}{\Rightarrow} \mathbf{B})_{3} \quad B_{3}^{2}-(\mathbf{E \times \mathbf { E }})_{3} \\
& \begin{array}{l}
\mathbf{P} \times \mathbf{Q} \stackrel{\operatorname{def}}{=} \overbrace{\left[\begin{array}{ccc}
\mathbf{i} & \mathbf{j} & \mathbf{k} \\
P_{x} & P_{y} & P_{z} \\
Q_{x} & Q_{y} & Q_{z}
\end{array}\right]}^{\operatorname{def}}=\mathbf{i}\left(P_{y} Q_{y}+P_{z} Q_{z}\right) \\
+\mathbf{j}\left(P_{x} Q_{x}+P_{z} Q_{z}\right)+\mathbf{k}\left(P_{x} Q_{x}+P_{y} Q_{y}\right)
\end{array}
\end{aligned}
$$

this multiplication of two vectors in Equation (12) may be called the parallel-vertical multiplication. The singularity (division by zero) in the prefactor $\frac{1}{\mathbf{E} \cdot \mathbf{B}}$ of the transformation matrix (10) is holding in the free space [7] and the singularity of the mentioned prefactor is avoided in a space of charge densities where the electric and magnetic fields are not necessarily perpendicular to each 
other.

To write the Lagrangian density for the Equations (7) or (8) in a form analogous to Equation (6) one needs to define a new vector field $Z_{\theta}$ such that

$$
K_{\theta v}=\partial_{\theta} Z_{v}-\partial_{v} Z_{\theta}
$$

In this way one can see that the following equations hold for $Z_{\theta}$

$$
\begin{gathered}
Z_{\theta}=\left(Z_{0},-Z_{1},-Z_{2},-Z_{3}\right) \\
\mathbf{Z}=\left(Z_{1}, Z_{2}, Z_{3}\right) \\
\nabla \times \mathbf{Z}=\mathbf{E} \\
\mathbf{B}=\frac{\partial \mathbf{Z}}{\partial t}-\nabla Z_{0}
\end{gathered}
$$

The form of Equation (8) suggests easily the following Lagrangian density

$$
\mathcal{L}_{2}=-\frac{1}{4} K^{\theta_{v}} K_{\theta v}-y_{v} Z^{v}
$$

which would give the Equation (8) as its equation of motion through the following Euler-Lagrange equation with the canonical coordinate being $Z^{v}$ and its derivative being $\partial^{\theta} Z^{v}$

$$
\frac{\partial \mathcal{L}_{2}}{\partial Z^{v}}-\partial^{\theta}\left(\frac{\partial \mathcal{L}_{2}}{\partial\left(\partial^{\theta} Z^{v}\right)}\right)=0
$$

The total Lagrangian density of the electromagnetic field would be the sum $\mathcal{L}_{1}+\mathcal{L}_{2}$, the expressions of the added terms of which can be taken, respectively, from Equation (6) and Equation (18), the two equations which have analogous forms, but having their differently defined respective vectors, namely, $A_{v}$ and $Z^{v}$ in addition to the difference in the upper and lower tensor indices.In taking the derivative of the second term on the left hand side of Equation (19), and obtaining Equation (8), the factor $1 / 4$ in Equation (18) is cancelled outduring the tensor algebra manipulations due to the Einstein summation convention that a repeated index in a multiplication of tensors implies a summation over the repeated index through all the conventional values of the concerned repeated index [8].

The electric field for a conductor or a semiconductor is proportional to the drift velocity and thus to the drift momentum as follows [1,5]

$$
\begin{aligned}
\mathbf{E} & =\rho \mathbf{J}=\rho \mathrm{nq} \mathbf{v}_{d}=\rho \mathrm{nq} \frac{c \mathbf{p}_{d}}{\sqrt{\mathbf{p}_{d}^{2}+m_{0}^{2} c^{2}}} \\
& =\rho \mathrm{nq} c \mathbf{p}_{d}\left[\left(\frac{\mathbf{p}_{d}^{2}}{m_{0}^{2} c^{2}}+1\right) m_{0}^{2} c^{2}\right]^{-1 / 2}
\end{aligned}
$$

When the following condition is valid

$$
\frac{\mathbf{p}_{d}^{2}}{m_{0}^{2} c^{2}} \ll 1
$$

Then using the approximation and the quantum mechanical operator $\mathbf{p} \rightarrow \frac{\hbar}{i} \vec{\nabla} \quad[9]$

$$
\mathbf{E} \approx \rho \mathrm{nq} \frac{\mathbf{p}_{d}}{m_{0}}\left(1-\frac{\mathbf{p}_{d}^{2}}{2 m_{0}^{2} c^{2}}\right) \approx \rho \mathrm{nq} \frac{\mathbf{p}_{d}}{m_{0}}=\frac{\rho \mathrm{nq} \hbar}{i m_{0}} \vec{\nabla}
$$

The $\mathbf{P}$ vector in Equation (12) may be replaced by the $\vec{\nabla}$ operator.

The quantity of the surface integral of the parallelvertical product can be reduced to a line integral around a curve in the counterclockwise direction analogous to Stokes' Theorem [4].

Let the parametric representation of a smooth differentiable surface be described by the following equation

$$
\mathbf{r}=X(u, v) \mathbf{i}+Y(u, v) \mathbf{j}+Z(u, v) \mathbf{k}
$$

Let the parallel-vertical product be written as follows where $\mathbf{Q}$ is a continuously differentiable vector field

$$
\begin{aligned}
& \overbrace{\left[\begin{array}{ccc}
\mathbf{i} & \mathbf{j} & \mathbf{k} \\
\frac{\partial}{\partial x} & \frac{\partial}{\partial y} & \frac{\partial}{\partial z} \\
Q_{x} & Q_{y} & Q_{z}
\end{array}\right]=\mathbf{d e f}\left(\frac{\partial}{\partial y} Q_{y}+\frac{\partial}{\partial z} Q_{z}\right)}^{\operatorname{din}\left(\frac{\partial}{\partial x} Q_{x}+\frac{\partial}{\partial z} Q_{z}\right)+\mathbf{k}\left(\frac{\partial}{\partial x} Q_{x}+\frac{\partial}{\partial y} Q_{y}\right)}
\end{aligned}
$$

The following vector product is a vector field perpendicular to the surface

$$
\frac{\partial \mathbf{r}}{\partial u} \times \frac{\partial \mathbf{r}}{\partial v}=\left|\begin{array}{ccc}
\mathbf{i} & \mathbf{j} & \mathbf{k} \\
\frac{\partial X}{\partial u} & \frac{\partial Y}{\partial u} & \frac{\partial Z}{\partial u} \\
\frac{\partial X}{\partial v} & \frac{\partial Y}{\partial v} & \frac{\partial Z}{\partial v}
\end{array}\right|
$$

And the unit vector perpendicular to the surface can be written as follows

$$
\mathbf{n}=\frac{\frac{\partial \mathbf{r}}{\partial u} \times \frac{\partial \mathbf{r}}{\partial v}}{\left\|\frac{\partial \mathbf{r}}{\partial u} \times \frac{\partial \mathbf{r}}{\partial v}\right\|}
$$

Then one can write the following mathematically stated theorem

Theorem: 


$$
\iint(\vec{\nabla} \times \widehat{Q}) \cdot \mathbf{n}\left\|\frac{\partial \mathbf{r}}{\partial u} \times \frac{\partial \mathbf{r}}{\partial v}\right\| d u d v=2 \oint\left(Q_{y} d z+Q_{z} d x+Q_{x} d y\right)-2 \oint\left(Q_{y} d x+Q_{z} d y+Q_{x} d z\right)
$$

Or:

\section{Proof:}

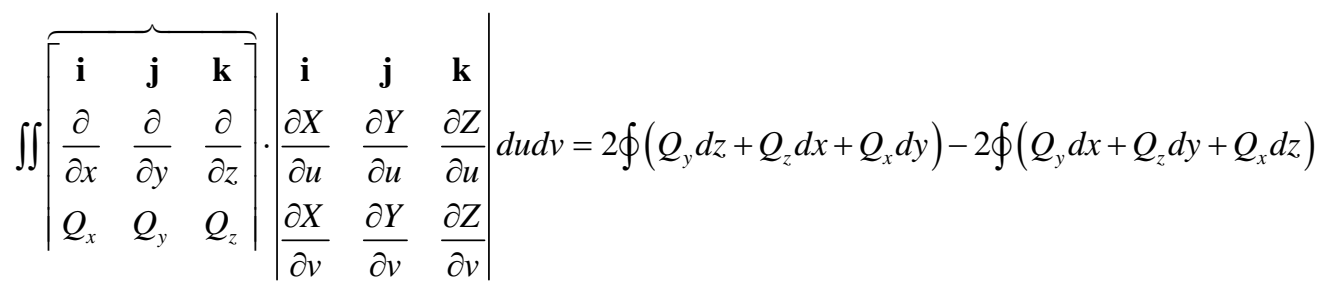

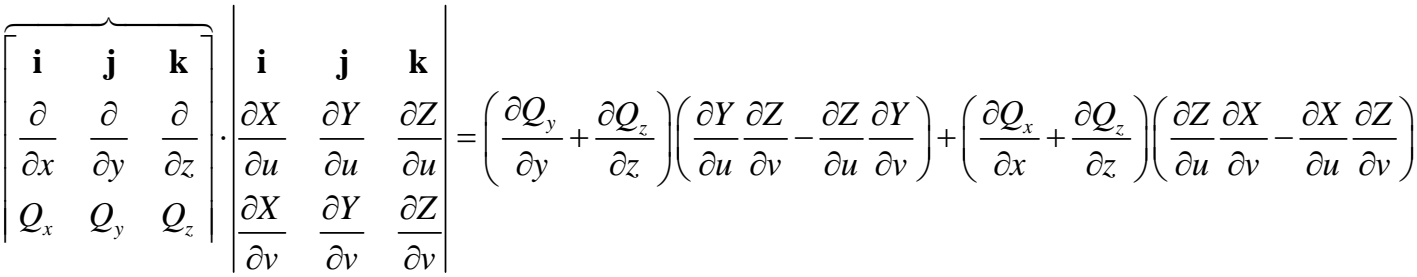

$$
\begin{aligned}
& +\left(\frac{\partial Q_{x}}{\partial x}+\frac{\partial Q_{y}}{\partial y}\right)\left(\frac{\partial X}{\partial u} \frac{\partial Y}{\partial v}-\frac{\partial Y}{\partial u} \frac{\partial X}{\partial v}\right) \\
& =\frac{\partial Q_{y}}{\partial y} \frac{\partial Y}{\partial u} \frac{\partial Z}{\partial v}-\frac{\partial Q_{y}}{\partial y} \frac{\partial Z}{\partial u} \frac{\partial Y}{\partial v}+\frac{\partial Q_{z}}{\partial z} \frac{\partial Y}{\partial u} \frac{\partial Z}{\partial v}-\frac{\partial Q_{z}}{\partial z} \frac{\partial Z}{\partial u} \frac{\partial Y}{\partial v}+\frac{\partial Q_{x}}{\partial x} \frac{\partial Z}{\partial u} \frac{\partial X}{\partial v}-\frac{\partial Q_{x}}{\partial x} \frac{\partial X}{\partial u} \frac{\partial Z}{\partial v} \\
& +\frac{\partial Q_{z}}{\partial z} \frac{\partial Z}{\partial u} \frac{\partial X}{\partial v}-\frac{\partial Q_{z}}{\partial z} \frac{\partial X}{\partial u} \frac{\partial Z}{\partial v}+\frac{\partial Q_{x}}{\partial x} \frac{\partial X}{\partial u} \frac{\partial Y}{\partial v}-\frac{\partial Q_{x}}{\partial x} \frac{\partial Y}{\partial u} \frac{\partial X}{\partial v}+\frac{\partial Q_{y}}{\partial y} \frac{\partial X}{\partial u} \frac{\partial Y}{\partial v}-\frac{\partial Q_{y}}{\partial y} \frac{\partial Y}{\partial u} \frac{\partial X}{\partial v} \\
& =\frac{\partial Q_{y}}{\partial y} \frac{\partial Y}{\partial u}\left(\frac{\partial Z}{\partial v}-\frac{\partial X}{\partial v}\right)+\frac{\partial Q_{y}}{\partial y} \frac{\partial Y}{\partial v}\left(\frac{\partial Z}{\partial u}-\frac{\partial X}{\partial u}\right)+\frac{\partial Q_{z}}{\partial z} \frac{\partial Z}{\partial v}\left(\frac{\partial Y}{\partial u}-\frac{\partial X}{\partial u}\right) \\
& +\frac{\partial Q_{z}}{\partial z} \frac{\partial Z}{\partial u}\left(\frac{\partial X}{\partial v}-\frac{\partial Y}{\partial v}\right)+\frac{\partial Q_{x}}{\partial x} \frac{\partial X}{\partial v}\left(\frac{\partial Z}{\partial u}-\frac{\partial Y}{\partial u}\right)+\frac{\partial Q_{x}}{\partial x} \frac{\partial X}{\partial u}\left(\frac{\partial Y}{\partial v}-\frac{\partial Z}{\partial v}\right) \\
& =\frac{\partial Q_{y}}{\partial u} \frac{\partial}{\partial v}(Z-X)+\frac{\partial Q_{y}}{\partial v} \frac{\partial}{\partial u}(X-Z)+\frac{\partial Q_{z}}{\partial v} \frac{\partial}{\partial u}(Y-X)+\frac{\partial Q_{z}}{\partial u} \frac{\partial}{\partial v}(X-Y)+\frac{\partial Q_{x}}{\partial v} \frac{\partial}{\partial u}(Z-Y)+\frac{\partial Q_{x}}{\partial u} \frac{\partial}{\partial v}(Y-Z) \\
& =\frac{\partial}{\partial u}\left[Q_{y} \frac{\partial}{\partial v}(Z-X)\right]-\frac{\partial}{\partial v}\left[Q_{y} \frac{\partial}{\partial u}(Z-X)\right]+\frac{\partial}{\partial u}\left[Q_{z} \frac{\partial}{\partial v}(X-Y)\right]-\frac{\partial}{\partial v}\left[Q_{z} \frac{\partial}{\partial u}(X-Y)\right] \\
& +\frac{\partial}{\partial u}\left[Q_{x} \frac{\partial}{\partial v}(V-Y)\right]-\frac{\partial}{\partial v}\left[Q_{x} \frac{\partial}{\partial u}(Y-Z)\right]
\end{aligned}
$$

Using Green’s Theorem [4] one can write

$$
\begin{aligned}
& \iint\left\{\frac{\partial}{\partial u}\left[Q_{y} \frac{\partial}{\partial v}(Z-X)\right]\right\}-\left\{\frac{\partial}{\partial v}\left[Q_{y} \frac{\partial}{\partial u}(Z-X)\right]\right\} d u d v+\iint\left\{\frac{\partial}{\partial u}\left[Q_{z} \frac{\partial}{\partial v}(X-Y)\right]\right\}-\left\{\frac{\partial}{\partial v}\left[Q_{z} \frac{\partial}{\partial u}(X-Y)\right]\right\} d u d v \\
& +\left\{\frac{\partial}{\partial u}\left[Q_{x} \frac{\partial}{\partial v}(V-Y)\right]\right\}-\left\{\frac{\partial}{\partial v}\left[Q_{x} \frac{\partial}{\partial u}(Y-Z)\right]\right\} d u d v=\oint\left[Q_{y} \frac{\partial}{\partial v}(Z-X)\right] d v+\oint\left[Q_{y} \frac{\partial}{\partial u}(Z-X)\right] d u \\
& +\oint\left[Q_{z} \frac{\partial}{\partial v}(X-Y)\right] d v+\oint\left[Q_{z} \frac{\partial}{\partial u}(X-Y)\right] d u+\oint\left[Q_{x} \frac{\partial}{\partial v}(Y-Z)\right] d v+\oint\left[Q_{x} \frac{\partial}{\partial u}(Y-Z)\right] d u \\
& =2 \oint Q_{y} d z-2 \oint Q_{y} d x+2 \oint Q_{z} d x-2 \oint Q_{z} d y+2 \oint Q_{x} d y-2 \oint Q_{x} d z \\
& =2 \oint\left\{Q_{y} d z+Q_{z} d x+Q_{x} d y\right\}-2 \oint\left\{Q_{y} d x+Q_{z} d y+\oint Q_{x} d z\right\}
\end{aligned}
$$


Which proves the Theorem.

\section{Conclusions}

The Lagrangian density of the electromagnetic field is complemented here by including a Lagrangian density for two of the symmetrized Maxwell's equations.In this procedure a transformation matrix is found which is including in its components two new definitions of vector products which are called here the "parallel-horizontal" and "parallel-vertical" vector products. The Theorem of the surface integral of the parallel-vertical vector product is shown to be reduced to a line integral.

\section{References}

[1] D. H. Young and R. A. Freedman, "University Physics," 9th Edition, Addison-Wesley Publishing Company, Inc., USA, 1996.

[2] W. E. Burcham and M. Jobes, "Nuclear and Particle Psy- sics,” Addison Wesley Longman Limited, Singapore, 1997.

[3] E. M. Purcell, "Electricity and Magnetism," Berkeley Physics Course, 2nd Edition, McGraw-Hill, Inc., USA, Vol. 2, 1985.

[4] T. M. Apostol, "Calculus," 2nd Edition, John Wiley \& Sons, Singapore, Vol. 2, 1969.

[5] J. B. Marion and S. T. Thornton, "Classical Dynamics of Particles and Systems," 4th Edition, Harcourt Brace \& Co., USA, 1995.

[6] I. V. Lindell, "Electromagnetic Wave Equation in Differential-Form Representation,” Progress in Electromagnetics Research, Vol. 54, 2005, pp. 321-333. doi:10.2528/PIER05021002

[7] G. R. Fowles, “Introduction to Modern Optics,” 2nd Edition, Dover Publications, Inc., New York, 1989.

[8] R. D’Inverno, “Introducing Einstein's Relativity,” Clarendon Press, Oxford, 1995.

[9] S. Gasiorowicz, "Quantum Physics,” 2nd Edition, John Wiley \& Sons, Inc., USA, 1996. 